Convergences francophones 6.1 (2019) : 18-32

http://mrujs.mtroyal.ca/index.php/cf/index

\title{
Le transgressif comme pratique textuelle politique dans l'œuvre de Rachid Mimouni
}

\author{
Dr. Azouz ALI AHMED \\ Queen's University
}

Je me propose, ici, d'analyser diverses modalités d'articulation du transgressif au politique dans la trilogie Le Fleuve détourné, Tombéza, L'Honneur de la tribu ${ }^{l} \mathrm{de}$ Rachid Mimouni, écrivain dont l'œuvre constitue un archétexte (Charaudeau et Maingueneau 60) eu égard à la somme des interrogations majeures et des commentaires qu'elle continue de susciter, mais aussi grâce à son potentiel symbolique virtuel de lecture dynamique et innovatrice du présent.

D'abord censurée au Maghreb, plus particulièrement en Algérie, son lieu d'ancrage sociétal, elle est, par la suite, en raison de son succès dans l'Hexagone, diffusée mais soumise à un déferlement de critiques dont la teneur discursive prend l'ampleur d'une véritable polémique : les médias ayant contribué à la transformer en un débat public largement orienté par les lignes éditoriales contrôlées par le pouvoir, où le discours littéraire ${ }^{2}$, de par sa posture paradoxique mesurée à l'aune de ses écarts par opposition aux normes d'une doxa consubstantielle à un modèle culturel hégémonique (au sens que lui confère Antonio Gramsci) et de la résonance de sa transgressivité généralisée, devient, comme dans une guerre idéologique et politique aux effets dévastateurs, l'ennemi à abattre par tous les moyens ${ }^{3}$.

D'ailleurs, il est utile de souligner à ce stade préliminaire de la réflexion que l'œuvre mimounienne s'est singularisée, à l'instar de bien d'autres d'écrivains contemporains d'Afrique du Nord tels Kateb Yacine, Boudjedra, Bourboune, Meddeb, Assia Djebar, Khair-Eddine, Choukri, Chraïbi et Khatibi avec une production essayistique d'une remarquable richesse théorique et critique ${ }^{4}$, mais de manière cependant autrement plus téméraire, provoquant une véritable rupture à la fois esthétique et thématique. Son œuvre s'impose comme contrepoint au grand récit linéaire d'une nation à la fois glorifiée et tétanisée « au moyen d'un discours incantatoire relevant de l'ordre du magique » (Danblon n.pag.). Ce discours este entendu comme procédé de persuasion appartenant à un registre rhétorique de

\footnotetext{
${ }^{1}$ Romans initialement publiés dans les années quatre-vingts par les éditions Laffont.

${ }^{2} \operatorname{logos}$; une parole néanmoins libérée dans les signes forts d'écrits que je m' autorise à classer comme performatifs.

${ }^{3}$ Il est capital de rappeler sur ce point que le discours du pouvoir est fortement marqué par un champ lexical propre, d'un impact psychologique étendu en raison du dispositif pragmatique déployé, à une situation conflictuelle permanente lorsqu'il se tient sur la défensive pour mieux justifier la répression et les atteintes constantes aux libertés. L'écrivain devient alors un danger à éloigner. Dans Le Vol du vampire. Notes de lectures, 90, Michel Tournier écrit : «Que peut-on craindre d'un écrivain quand on dispose d'une police, d'une armée, de juges vendus et de prisons ? C'est sans doute que le pouvoir ne repose jamais sur la seule force matérielle, mais qu'il n'est rien sans un ensemble d'artifices psychologiques destinés à emporter le consentement moral des 'sujets'. Or c'est ce consentement plus ou moins extorqué et les artifices qui l'entretiennent que l'écrivain vient ébranler par ses seuls écrits. »

${ }^{4}$ Citons comme exemple La Blessure du nom propre.
} 
construction d'une autorité absolue, dans le champ littéraire maghrébin de l'époque, si l'on considère les thèmes toujours actuels, dont l'acuité demeure toujours aussi vive, qui structurent l'ensemble de la trame romanesque mimounienne : sexe, viol, religion, histoire, zoophilie, inceste, bâtardise, déviances multiformes, oppression des femmes, gouvernance, corruption, etc. Autant de sujets tabous et de forte contestation, même feutrée, nullement réduite en dépit de sévères mesures policières, surtout dans une société où l'absence de démocratie ouvre le champ aux dérives du pouvoir, de ses nombreux relais, avec, entre autres, les institutions religieuses en charge de la moralisation des masses. En outre, toutes les questions brûlantes, ainsi que les choix orchestrés par ceux qui gouvernent sont de facto évacués de l'espace public. Car, conforté dans sa vocation critique plurivocale, ce dernier susciterait inévitablement des débats sur le système de gouvernance et pourrait ébranler un tant soit peu le socle civilisationnel dont l'islam demeure le référent fondamental. Celui-ci est cependant soumis à des interprétations variables en fonction d'intérêts l'éloignant sans équivoque d'une relecture herméneutique non contrainte, rationnelle de par son ouverture à d'autres dimensions de l'expérience du monde. On retrouve cette relecture çà et là, surtout en Occident, où elle circule sans trop d'entraves, éclairant de nouveaux faisceaux l'inscription $a b$ ovo du Coran, en tant que message universel, dans la modernité : citons entre autres Mohamed Iqbal, Mohamed Arkoun et Malek Chebel. Autrement dit, la politique dans sa dimension sociale construite sur « un fait : la pluralité humaine » (Arendt 95) n'a point de voies d'expression, ni de représentation légale ni même de chapitre à proposer à l'ordre du jour d'un présent scellé. Ce qui forcément la pousse à en emprunter d'autres comme alternatives transformées par le levain du subversif, recherchées dans le mouvement perpétuellement déstabilisateur de l'espace composite des marges, parfois inconscientes, surgies des limbes de l'imaginaire social, ou imposées par les contradictions qui travaillent en profondeur la société. Mais aussi dans la panoplie des formes oppositionnelles mettant en branle et, subséquemment, en relief les capacités d'invention et de création que manifeste par là même le corps social. Toutes ces formes d'action rebelles ont comme levier d'Archimède un fonds culturel immémorial toujours vivant et producteur de sens, à l'intérieur même du système en tant qu' " ensemble de pratiques et d'institutions » (Le Petit Robert micro 1392). Ces formes contournent le système tout en affirmant ${ }^{5}$ l'existence d'individus qui appartiennent à des collectivités, insoumises dans la mesure où leur combat multiforme leur permet d'échapper à la domination totale qui s'exerce au moyen de la panoplie de dispositifs structurant l'espace sociopolitique et économique, et porteuses de valeurs et de visions du monde différentes.

Assise sur ce cadre heuristique et épistémologique esquissé à grands traits, la problématique introduite ci-dessus va permettre, dans un premier temps, de situer, à travers leur questionnement, l'évolution, comme modes opératoires de saisie du réel, des notions clés structurant notre analyse de la trilogie mimounienne. Dans un deuxième temps, j'exposerai comment les multiples tensions qui sous-

\footnotetext{
${ }^{5}$ Voir de Certeau 1980.
} 
tendent l'univers romanesque de Mimouni se configurent en lignes de fractures matricielles. Enfin, soumettant la dimension théorique, en une sorte de chassécroisé, à l'épreuve du matériau fictionnel, je tenterai une saisie de la dynamique socio-historique et culturelle qui, dans les trois compositions romanesques, met en jeu les différentes représentations qui informent l'articulation complexe du «transgressif » au « politique ».

\section{Notions polysémiques, fluctuantes et en devenir}

De nombreux travaux, en constant renouvellement, traversent les champs de la théorie et de la critique littéraire, de même ceux des sciences sociales, juridiques, économiques, politiques, et continuent à porter un vif intérêt aux notions - transgression et politique - diamétralement opposées, si l'on considère les présuppositions qui les entourent. Ils attestent de la vitalité de celles-ci qui, loin de revêtir les effets délavés de topoi avachis, gardent une pertinence, jamais démentie, consubstantielle à l'évolution de la pratique littéraire, dont les poussées représentent autant d'avancées que de ruptures. Le mouvement de la modernité en atteste de manière éloquente par sa sensible distanciation, avec Hugo et Musset, du théâtre classique, et de la poésie que vont bouleverser radicalement, dans le même élan novateur, esthétiquement transgressif, un peu plus tard, en tant que genre, Baudelaire et Rimbaud. Tout comme des conventions du réalisme, dans la deuxième moitié du XIX ${ }^{\mathrm{e}}$ siècle, que Flaubert marque de son empreinte finement ironique et désacralisante. Une modernité qui se traduit comme un puissant phénomène social, c'est-à-dire «un art de faire profondément enraciné dans le quotidien » (de Certeau 80), mais aussi, plus précisément, comme un mouvement de la société globale, que je définis comme une «totalité dynamique en flux perpétuel », que seule une pensée complexe peut saisir (comprendre, expliquer, interpréter) en raison de ses innombrables formes d'organisation, de conflits et de ses jeux et/ou enjeux de pouvoir dont l'arbitrage, souvent ardu, aux conséquences parfois imprévisibles, est dévolu à l'État. Je dirais au politique dans un sens large, car l'État ne se définit pas exclusivement, si ce n'est dans ses prolongements constitutionnels, par «la politique », dans les régimes démocratiques ou autres d'où, dans une certaine mesure, la fission, productive à maints égards d'ailleurs, de la définition première du concept parce qu'impuissant à rendre une image claire des rapports de pouvoir dans leur complexification, mais aussi, et surtout dans leur mise en discours critique. C'est à partir de cette perspective ouverte que je qualifie, comme beaucoup d'autres le tentèrent dans des études accomplies, les signifiants «transgression» et «politique » de notions (polysémiques) qui informent les configurations nouvelles et mouvantes de la réalité, et non de concepts qui limitent dès lors que définis, ou appellent de nouvelles définitions toutes aussi contraignantes les unes que les autres. ${ }^{6}$ Ces notions, quelquefois, nous amènent

${ }^{6}$ Cette réalité est néanmoins inscrite dans une temporalité-durée-historicité, dont l'œuvre romanesque s'empare subtilement, même avec des ratés, pour la reconstruire au profit de son propre univers qui n'en constitue nullement un miroir déformant, produit de l'idéologie, mais beaucoup plus une saisie de ses multiples formes d'aliénation et de ses apories. 
cependant à nous questionner sur la justesse de nos propres observations. En effet, la variabilité du transgressif fait qu'il peut cesser de l'être sur des questions socioculturelles et politiques aux tensions réduites de par le travail du temps et l'action corrosive que subissent les forces contraires au changement sous toutes leurs formes et masques, sans toutefois lâcher prise sur d'autres moins visibles, et passer dans l'ordre du normatif dans des sociétés où l'on s'y attend le moins. Les œuvres de Khayyam, Cheikh El Nafzaoui, Sade, Bataille, sont, aujourd'hui, interrogées à partir de prismes différents, même sur leur contenu transgressif au parfum d'essences scandaleuses. Qu'il suffise de même de rappeler, pour être au plus près de l'histoire récente, que l'abolition de la polygamie (1957) et le droit à l'avortement (1973) en Tunisie ${ }^{7}$, furent consacrés, au lendemain de l'indépendance obtenue en 1956, fruits d'une grande réforme, que d'aucuns auraient jugée comme prématurée alors qu'elle ouvrait la voie, en un moment historique fondateur, à l'émancipation des femmes, prenant ainsi de vitesse la fermeture du champ politique et le retour précipité du conservatisme, pour ne pas dire révolution dans un pays musulman où les écarts par rapport au «canon » relèvent d'une délicate audace, même dans l'anonymat du geste rebelle, ou d'un courage politique exceptionnel.

La notion de transgression vient du latin transgressio (marcher à travers, au-delà); transgresser, c'est aussi enfreindre, être contraire à, violer (acte de violer une loi), désobéir, passer outre un interdit, mais cela signifie également « donner à voir et à penser autrement ». La transgression, pour reprendre Dominique Berthet, « est une posture critique », mais aussi un moyen de résistance qui, comme l'œuvre mimounienne le montre, fait converger elliptiquement les regards vers d'autres horizons. J'y reviendrai plus avant.

D’emblée, de façon nuancée, rappelons que la notion de «politique » est protéiforme à maints égards. Qu'elle pose problème du fait de son usage abusif, parfois réducteur lorsqu'elle se limite, à dessein, au caricatural. Mais il n'échappe à personne, de nos jours, que «le politique » précède «la politique ». En effet, les sociétés humaines se découvrent, dès les premières traces de vie communautaire et d'organisation de l'espace social, de leur passé anthropologique, comme des entités politiques. Les Grecs, qui inventent la notion (polis), l'attribuent à tout ce qui touche au gouvernement de la cité : une définition aristotélicienne du politique par l'État, dont les sociétés modernes, sommées par de nouveaux impératifs, s'éloigneront peu à peu. Le philosophe Maurice Gauchet, auteur de Désenchantement du monde et Condition politique, redonne au politique, en reprenant Aristote, à travers la notion de $l^{\prime}$ « Être-collectif» une place centrale comme outil de gouvernance de la collectivité humaine. Il pense même que le mariage entre «la politique » et «le politique», devenu une exigence dans la société moderne, doit être consommé pour impulser un souffle nouveau à l'art de se gouverner. Ce positionnement est évidemment battu en brèche par le néolibéralisme (qui vassalise les États, et les peuples par ricochet, devenus les commis du Capital). Gauchet ne l'ignore pas quand il explique sa totale suprématie,

\footnotetext{
${ }^{7}$ Alors que la Loi Veil l'autorisant n'a été votée qu'en 1975 au Palais Bourbon.
} 
et la juge irréversible. Pour lui, son objectif principal est de transformer, à l'insu des citoyens, souvent obligés de se soumettre aux lois votées en dehors des parlements nationaux, le monde en un vaste marché ouvert aux multinationales, inaugurant de la sorte une nouvelle ère de l'impérialisme. Un impérialisme hybride, aux identités multiples que je définirai comme «multilatéral » si l'on considère le déclin de certaines grandes puissances, l'émergence de nouvelles, ou le retour d'anciennes sur la scène du monde.

Par ailleurs, la prolifération des définitions du politique (Le Littré, à lui seul, en donne huit) va complexifier, l'évolution de l'organisation sociale oblige, la réflexion sur les sujets reliés à la source et à l'exercice du pouvoir. Aussi, dans le cadre de cette étude, je retiendrai celle qu'en donne Ardoino, tout en gardant une certaine distance critique vu que mon attention porte sur l'articulation (c'est-à-dire sur les liens qui se nouent, se créent, s'intensifient, s'enchevêtrent) du transgressif au politique dans un univers romanesque mimounien où la politique n'existe que sous forme de leurres : images virtuelles d'un système fermé ${ }^{8}$. Et où le transgressif comme pratique textuelle, tout en épousant toutes les formes de rupture (esthétiques et autres), engendre en quelque sorte «le politique » comme substitut à la vacance de «la politique » à faire ad(venir) comme lieu de négociations, d'arbitrage consensuel, de justice et de développement des rapports humains à l'intérieur d'un même espace géographique (pays, État) ou dans le cadre de relations interétatiques (engageant les nations formant l'ensemble des êtres qui peuplent la planète).Il participe donc à la mise en question, à travers la société du roman, d'un monde celui du cotexte - où se joue une tragédie insensée. C'est ainsi que pour Ardoino :

On peut distinguer entre trois formes substantives : les politiques (féminin pluriel) élaborées dans le cadre des organisations, notamment des entreprises (commerciales, industrielle, financière, de recherche, de fabrication, du personnel...) correspondant aux grandes «fonctions » de l'administration (Henri Fayol) et de la gestion (les Anglais opposent ainsi policies à politics); la politique regroupant les institutions, les agents et les acteurs de la vie politique dans le cadre d'une démocratie représentative ; le politique plus inspiré par les conceptions gauchistes d'une démocratie directe où le citoyen se veut sujet politique, conscient de ses droits et riche d'une conscience critique. Cette constellation de termes intéresse donc principalement la dévolution démocratique et l'exercice du pouvoir qu'on espère, à l'échelle macrosociale, dialectisé par le jeu des rapports de forces et contrôlé par les instances commises à cet effet. (29)

\section{Lignes de fractures matricielles}

Par projection rétroactive, et pour situer, dans une vision englobante, permettant de mettre en relief la macrostructure sémantique de chacun des romans de la trilogie et l'articulation du transgressif au politique, nous dirons que Le Fleuve

${ }^{8}$ Deux auteurs abordent cette problématique dans des essais critiques importants : Rédouane, Nagib, Lecture(s) de l'œuvre de Rachid Mimouni. Paris : L'Harmattan, 2012 ; Gafaïti, Hafid, La Diasporisation de la littérature Post-coloniale. Assia Djebar. Rachid Mimouni. Paris: L'Harmattan, 2005. 
détourné, première œuvre de la trilogie, dont la scénographie repose sur un dispositif carcéral met en scène l'échec de la révolution. En effet, Mimouni fait tuer symboliquement la liberté ${ }^{9}$ par le personnage principal, un ancien combattant (Moudjahed en arabe algérien) et exprime ainsi la métamorphose transgressive de la révolution dont les idéaux tombent comme un château de cartes. Les mésaventures du protagoniste, un héros-revenant auquel on a suggéré avant son incarcération, à force de menaces à peine voilées, de garder son statut de fantôme afin de ne pas perturber l'ordre établi, et de ses compagnons de prison (tous des transgresseurs) mettent en lumière cette métamorphose dans un procès narratif assez complexe (constants décrochages), suspendu et flottant entre le présent et le passé. Ce procès se compose de «convulsions » de récits intercalés que rythme l'instance narrative tournante composée du héros (au récit discontinu le plus long) et de ses camarades de détention, les plus proches de lui : Omar, Fly-Tox, Vingtcinq, l'Écrivain. C'est aussi pourquoi la numérotation ne s'inscrit pas dans une suite chronologique normale, mais en un découpage en parties non indiquées clairement (on passe par exemple du chapitre 22 à un nouveau chapitre 1, et ainsi de suite). Il est possible, à ce niveau, de parler de rupture esthétique, donc de transgression par rapport aux conventions romanesques classiques. Dans cette œuvre, les chapitres correspondent à des séquences temporelles qui se chevauchent, un peu comme dans des mini-récits concomitants, et se succèdent à la fois tout en accentuant le brouillage des indices narratifs identificateurs. Le narrateur-personnage principal, autour duquel se déroule l'histoire, nous pousse à sa guise, au moment où il le juge opportun, dans les méandres labyrinthiques de son récit : ceux du présent dont il mesure la gravité et ceux du passé encore douloureux et obsédant malgré les moments de bonheur et d'équilibre antérieurement vécus, avec une femme épousée malgré l'opprobre qui pesait sur elle, ou au maquis avec des compagnons solidaires. Ainsi s'installe ce que je définis comme une «dynamique temporelle » qui efface les barrières du temps et de l'espace.

Quant au deuxième roman, Tombéza, c'est d'abord son titre énigmatique et effrayant qui suscite des interrogations. «Tombéza», nom du personnage principal, est un mot impropre, sans signification précise, et construit par dérivation du lexème «tombe ». Ces questionnements prennent forme à travers la narration d'une odyssée au cœur d'une société en déréliction totale, où le transgressif se généralise, dans une tension permanente entre le négatif et le positif et touche l'ensemble des couches sociales. Ainsi, l'échec (ou fracture) matriciel de la révolution qui configure les multiples récits du Fleuve détourné se transforme en ce que j'appelle un «cauchemar éveillé » dans Tombéza où se peint, à petites touches, comme sur une toile de couleurs sombres aux nuances imperceptibles, la désagrégation de l'ensemble des valeurs morales et sociales (seconde fracture matricielle), autrement dit la révolution a perdu son sens et sa boussole. Tout est inversé, ce que suggère imperceptiblement un usage itératif de l'hyperbate dans ses nombreuses configurations. De plus, le visage de Tombéza, cauchemardesque en

\footnotetext{
${ }^{9}$ Pour, par ellipse, interroger sur son absence.
} 
raison d'une déformation congénitale ${ }^{10}$ est un renvoi constant au réel : celui que l'on cherche à cacher, à fuir ou à illusoirement accepter en l'expliquant par toutes sortes de déterminations qui alimentent les discours incantatoires que les religieux répètent à l'envi faisant croire que l'on conjure ainsi, en dehors de toute temporalité, le sort qui frappe durement le pays et ses habitants.

L'honneur de la tribu qui clôt la trilogie raconte l'impossible mariage entre la tradition et une modernité inaccomplie en raison à la fois de la trahison de la révolution par des faussaires à travers les errements des dirigeants ${ }^{11}$ et des hauts fonctionnaires de l'État qui s'obstinent à construire le socialisme, auquel ils ne croient point, de façon autoritaire sans consulter le peuple, véritable héros de la tragédie coloniale. A cela s'ajoute le conservatisme qui fige la tradition dans ses aspects les plus rétrogrades. La négociation d'une ouverture socioculturelle orientée vers le futur pérenniserait les valeurs ancestrales, dont les caractéristiques fondamentales mettent plus l'accent sur la cohésion sociale en termes d'intégration et de partage équitable des ressources entre les membres de la communauté et non de manière particulière sur le religieux qui tient son rôle moral et spirituel dans la stricte observance de ses limites, comme socle solide d'une nation aux membres partageant depuis des siècles le même destin. Autre faille (ou fracture) au plan idéologique. Ainsi la tradition se perd car son héritage est mal fructifié et la modernité déçoit, voire fait régresser au plan sociopolitique, parce qu'elle se trouve entre des mains qui la détournent de l'esprit émancipateur que lui ont légué les Lumières. Et que la révolution a fait entrevoir.

Afin de situer de manière un peu plus prononcée les lignes de fractures matricielles, je résume les événements marquants qui structurent la genèse des trois romans par le tryptique : cosmos, anthropos et logos. En effet, Le Fleuve détourné, titre thématique et polysémique renvoie à la défiguration de l'espace physique qui prend forme en tant que matériau descriptif dans le texte : construction d'un barrage par des ingénieurs étrangers. De plus il agit comme une métaphore assez puissante de la révolution algérienne interrompue au lendemain de l'indépendance ; ce qui signifie indirectement que le système mis en place asphyxie toute vie politique. Le viol de l'environnement fonctionne de même comme préfiguration au viol répété de Houria, la femme du révolutionnaire (elle sera séquestrée par des fonctionnaires véreux qui en feront un objet de plaisir, une esclave sexuelle). Le viol de l'espace physique (cosmos), l'occupation du territoire (et de l'assujettissement de ses habitants) fait mène au viol de l'espace corporel (relevant de l'anthropos) qui est aussi symbolique dans le cas de Houria dont le nom signifie en arabe algérien « liberté », qui, selon Baechler :

Se définit par opposition à trois contraires distincts. Elle s'oppose à l'oppression pour revendiquer la pluralité des choix ; à la coercition pour affirmer l'autonomie ;

\footnotetext{
${ }^{10}$ Cette déformation est due aux effets secondaires du traitement inhumain subi par sa mère juste après son agression physique qui la rendra impotente et par les vaines tentatives d'avortement qui suivront.

${ }^{11}$ Pour la plupart absents durant la guerre de libération nationale des lieux où se livraient les batailles contre l'ennemi.
} 
à la sujétion pour viser l'association ou la participation. Au niveau individuel, on retrouve une aspiration à ces trois formes. (205)

D'autre part, dans Tombéza, usant de la gradation au niveau microsocial, Mimouni donne à l'expression de la violence une allure terrifiante. La jeune femme, future mère de Tombéza, violée, sera complètement défigurée par les coups de canne de son père et physiquement anéantie, ne pouvant même plus utiliser normalement les fonctions biologiques de son corps : manger, uriner et déféquer proprement, vaquer à des occupations domestiques ou autres. En outre, Tombéza nous plonge au sein de l'univers d'un homme sans avenir qui survit dans un monde sans repères où il s'associe avec un commissaire de police corrompu pour commettre les pires méfaits. Cette complicité mafieuse revêt un caractère anticipatoire si l'on considère certaines données relatives à la corruption que livre la presse internationale au sujet de la gouvernance de l'État algérien d'aujourd'hui miné par l'alliance du monde des affaires et de la classe dirigeante issue ou héritière de l'ancienne bourgeoisie d'État.

D'un autre côté, la gradation opère comme un raccord à triple entrées d'une grande justesse entre les trois œuvres : Le Fleuve détourné représente de prime abord un hologramme des futurs préjudices que subira le pays en tant qu'espace social ; Tombéza, à un niveau plus élevé, cerne la dégradation de l'individu à travers le relâchement des rapports sociaux et les misérables conditions de vie de la majorité dominée et elle-même corrompue pour survivre : une véritable descente aux enfers. Quant à L'Honneur de la tribu, il consacre la perte des valeurs, c'est-àdire le capital symbolique qui permet à une société de résister aux plus terribles des tempêtes ou des fléaux : les grandes invasions et les périodes coloniales, tout comme les crises morales et politiques qui secouèrent la nation et menacèrent son existence, comme celles de l'été 62 et d'octobre 88 par exemple. C'est pourquoi ce dernier roman nous situe dans l'espace du discours, du conflit des valeurs et de la confrontation idéologique. Le logos (la parole, le discours, la rhétorique ou art de la persuasion) qui devrait en principe permettre aux hommes d'évoluer dans des espaces de liberté, de trouver des moyens politiques pour vivre ensemble dans un monde que symbolise le cosmos, ne fonctionne, dans l'univers romanesque mimounien, que dans un sens unique, celui qui impose d'autorité l'assujettissement de la majorité. Et c'est le transgressif qui va en quelque sorte faire bouger les lignes même à travers ce qui pourrait s'interpréter comme charge de négativité. Mais celle-ci remet tout à plat, soumet au questionnement, introduit de nouveaux rapports et impose une forme de vie politique, même non reconnue officiellement. Tout ce qui trouble les normes sociétales imposées par la loi appelle néanmoins une réponse de la part du pouvoir, donc l'oblige à réprimer ou à chercher des compromis par la négociation dont les termes sont souvent imposés. Dans Le Fleuve détourné, la négociation entamée lors de la grève des éboueurs n'aboutit à rien si ce n'est qu'elle a pu ouvrir durant un laps de temps le champ politique, montrer que les cols bleus existent, qu'ils peuvent faire un peu peur même si leur situation sociale ne change pas, tout comme leurs rapports avec l'administration qui ne cédera aucune parcelle de pouvoir ni ne répondra favorablement à la moindre de leurs revendications. Cela 
constituerait, pour elle, un aveu de faiblesse et non une gestion rationnelle des conflits de travail par le dialogue et la concertation avec les grévistes ou d'autres segments de la société vivant des crises dues à la mauvaise prise en charge par l'État de ses responsabilités. Cet épisode, riche en enseignements, permet à Mimouni de reposer indirectement la question fondamentale de la démocratie, de la politique, c'est-à-dire du pouvoir par représentation : existence de partis politiques rivaux, de médias libres, etc., qui ne sont pas cependant, il faut le préciser, une garantie absolue, en particulier dans les pays où le système politique repose sur le néopatrimonialisme étatique. Ainsi, le transgressif - faire grève en contournant la loi qui les interdit - devient, comme en démocratie directe et citoyenne «le politique » qui interpelle sur des problèmes majeurs de représentativité, de gouvernance, de gestion et d'organisation qui, dans les faits, soulignent l'absence de « la politique ».

\section{Lieux de convergence et d'articulation}

Claude Duchet souligne l'importance des liens qui existent entre le titre d'un roman et son texte, tout comme leur indissociable et féconde complémentarité. En effet, le titre représente une matrice-programme et donne un éclairage même partiel à la fois sur la forme et le contenu du roman ou de l'œuvre en question. Aussi, l'analyse montre que les titres des romans qui composent la trilogie mimounienne sont des lieux, des ensemble de signes où s'énonce le transgressif. Le verbe détourner, adjectivé dans Le Fleuve détourné est un synonyme de transgresser; Tombéza, terme construit à partir du mot «tombe » et aussi du verbe «tomber » ou faire tomber, renvoie de même à une distorsion, à un enfermement, ou à une chute provoquée. Les deux titres prennent forme dans l'imaginaire comme images vives d'une représentation d'un événement passé ou futur. La transgression s'articule en une projection coextensive du sens au politique. En effet, qu'est-ce qu'un fleuve détourné ? La dimension métaphorique renvoie, la duplicité du discours romanesque le montre ingénieusement, au pays devenu indépendant et libre que les dirigeants métamorphosent en une véritable prison : le narrateur et ses compagnons énoncent à partir d'un lieu de détention qui, par synecdoque généralisante, s'élargit à l'ensemble du pays, devenu un espace de non-droit. De plus, on remarque la transgression écologique qui va permettre un déplacement vers l'ordre symbolique (les signes, le discours), d'une part, et politique, d'autre part, au regard des conséquences à la fois sur l'environnement et sur l'organisation de la vie sociale, c'est-à-dire l'espace du politique dans un de ses nombreux aspects. Le fleuve dont il est question est détourné par des travaux suite à un plan de réaménagement du territoire conçu par des ingénieurs venus d'ailleurs, c'est-à-dire, et même si ce n'est pas dit clairement, du camp socialiste. Effectivement, quelques chapitres évoquent les dirigeants soviétiques qui serviront de prête-noms aux leaders algériens nommément absents: ce qui met davantage en relief les choix politiques autoritaires de ces derniers dans la mesure ou la comparaison implicite agit comme un leurre et peut voiler quelque peu la critique. Le deuxième roman, dont le titre Tombéza surprend à maints égards, montre que le transgressif peut revêtir une dimension onomastique, voire anthropologique et parfois religieuse, portée par un 
Convergences francophones 6.1 (2019) : 18-32

http://mrujs.mtroyal.ca/index.php/cf/index

personnage central dans une œuvre au titre-éponyme. Un sobriquet donné par des enfants à un bâtard difforme ayant un rictus qui, à lui seul, comme monstration exprime toute l'horreur de l'existence plastique : en arabe populaire maghrébin, on dirait $m$ 'tabaz. Tombéza serait donc une personne revenue de la mort (qui a emporté sa mère en couches) ou qui ne mériterait pas de vivre en tant que bâtard, mais qui va déterrer beaucoup de choses de la tombe de l'oubli, mettre à nu les tares de la société. Il dit à un moment où il rappelle comment sa mère a été sauvagement battue par son propre père :

La fornication! hypocrite société ! Comme si je ne savais pas ce que cachent tes apparences de vertu, tes pudibonderies, tes tartufferies. Mille et mille intrigues des tourments de la chair. Les messes noires et les rites d'envoûtement. J'ai su le frère qui engrossait la sœur, pour étouffer ensuite entre ses mains le nouveau-né criard, j'ai su la femme à la parfaite réputation qui se donnait au fond du jardin à des inconnus, à des mendiants, à des enfants impubères, j'ai su les cocufiages sournois entre frères, les hommes stériles qui supposent leur femme inféconde et l'envoient vers des guérisseurs, sans doute pour les effets de leur virilité... Alors une fillette de quinze ans encore à s'effrayer de ses premières règles et qu'on écartèle auprès d'une source. Elle n'a su que clamer son malheur, la niaise, normal que celles-là s'acharnent sur celle-ci. Par son drame révélé au grand jour, elle expiera pour toutes les autres. Pauvre fille ! Pauvre femme ! Votre malédiction, c'est cette membrane qui ferme votre vagin. Quelle obscure mais implacable nécessité a conduit la nature à vous doter de ce catastrophique hymen? Tout aurait été si simple sans cela. Un bout de chair turgescent qui pénètre dans une cavité de chair, pas de quoi faire un drame. Un bon bain suffirait à effacer les traces de mille viols successifs. Une petite membrane qui fonde une civilisation. (34-35)

Le transgressif, dans cette citation, malgré le long développement de l'isotopie sexuelle, opère à plusieurs niveaux discursifs. Le héros-narrateur, un bâtard c'està-dire un enfant né hors mariage dans une société puritaine, donc condamné, de surcroît, même en tant que victime à une vie de paria, dénonce la situation subie par sa mère et surtout l'hypocrisie qui entoure la problématique du sexe. Celui-ci ne s'évoque que symboliquement en public, avec des précautions langagières dont les normes relèvent de textes religieux, mais aussi de traditions socioculturelles imposant des règles de bienséance en société : dans le Coran, les organes génitaux ne sont dits qu'allusivement et par symboles même si la sexualité, évoquée dans 82 sourates qui circonscrivent son cadre légal, est fortement recommandée comme source d'équilibre conjugal et de bonheur. Ce sont les interdits (de l'ordre du transgressif) qui créent et multiplient les déviances : inceste, cocufiage entre parents, infanticide, recours aux pratiques magiques, etc. Ces tabous ne permettent pas de promouvoir des relations adéquates, saines, basées sur les principes d'un projet de société aux rapports égalitaires en droits entre hommes et femmes, et contribuent partant à maintenir l'ignorance, source de sous-développement et de stagnation. L'ensemble des tares que le narrateur oppose de manière discursive au milieu, frappé d'un malaise qui appellerait sans contredit une lecture psychanalytique, dont il est issu malgré son statut (relevant d'une identité 
paratopique), renvoie à la condition faite aux femmes prisonnières de pratiques coutumières de domination masculine qui font de leur corps, aux attributs d'une immense richesse que les sciences thésaurisent, non pas un moyen d'émancipation mais d'enfermement et de soumission, sous la menace permanente d'agressions sexuelles et de viol. La dernière phrase de la citation «Une membrane qui fonde une civilisation » bâtie sur un oxymore ${ }^{12}$ recèle une puissante et transgressive ironie par le biais de laquelle le scripteur, faisant une irruption intempestive dans le texte, s'adresse aux forces de l'inertie, du conservatisme et du pouvoir et semble leur dire que la civilisation, aujourd'hui, se construit sur d'autres paramètres : éducation, liberté, sciences, démocratie, respect des droits humains.

Dans L'Honneur de la tribu, Mimouni montre comment l'entreprise de destruction ou de déracinement va toucher même les lieux de repli ou de sauvegarde de la société traditionnelle perchée sur un piton de rocher dans un douar de fin du monde. L'honneur, valeur surdéterminante du point de vue doxique dans les sociétés méditerranéennes sera bafoué par le propre enfant de la tribu et transformé en humiliations de toutes sortes sous prétexte de modernisation. En effet, Omar El Mabrouk, un ancien forban qui avait engrossé sa propre sœur, pris le maquis durant la révolution et est devenu un haut fonctionnaire chargé de créer une nouvelle entité administrative, une préfecture (Wilaya en arabe algérien) :

Jours après jours les engins étrangers modifiaient notre paysage. Lorsqu'on projeta de dynamiter nos oliviers, Omar El Mabrouk vint nous voir.

- Il n'y a pas lieu d'en faire un drame. Ces cupides arbustes ne vous rendaient qu'en fruits étiques votre large peine. De plus l'huilerie du boiteux étant fermée, je vois mal ce que vous pourriez faire de vos récoltes. En tout cas, vous serez généreusement indemnisés ...

- Mais de quoi vivrons-nous ?

- N'ayez crainte. J'y ai pensé. Je vous promets qu'ici, en ce pays du bout du monde, je ferai venir des machines qui perceront la glaise et le schiste pour aller au plus profond chercher et faire jaillir l'eau en sa prime force. Vous pourrez alors cultiver la pastèque si douce, le cantalou odorant, le piment de feu, l'haricot fragile, la tomante exigeante, la poire au goût plus succulent que les lèvres de votre première amante. Vous deviendrez riches et gras.

- Nous étions à quelques jours de la récolte, mais les gendarmes d'Omar El Mabrouk refusèrent de nous laisser pénétrer dans les champs ...

Je crois que c'est à partir de ce jour que nous commençames à prendre conscience des ravages causés par l'arrivée d'Omar El Mabrouk. (168-170)

Omar El Mabrouk, dont le nom renvoie à un des quatre khalifes qui succédèrent au prophète Mohammed, se traduit onomastiquement comme l'élu, porteur de la félicité. Or l'ironie subtilement dévastatrice de Mimouni montre que c'est lui qui incarne la transgression dans sa négativité la plus significative et donne à la « pensée magique », que j'ai cernée, en introduction, comme étant un des éléments de construction de l'autorité absolue, toute sa dimension. Omar El Mabrouk détruit

\footnotetext{
${ }^{12}$ La fragilité d'un hymen que le narrateur désigne par un terme propre au lexique médical, donc
} scientifique, et la solidité d'une civilisation qui dure des siècles. 
« l'existant » (l'agriculture vivrière) de la société traditionnelle à laquelle il invente un futur hypothétique, fruit de son imaginaire de fonctionnaire détenteur d'une autorité sans limites. C'est pourquoi personne aujourd'hui ne conteste le fait que l'Algérie vit une véritable catastrophe écologique qui lamine inéluctablement son potentiel agricole : accaparement des meilleurs terres du littoral pour des projets immobiliers ou industriels, constructions anarchiques, perversion des modes alimentaires traditionnels qui restreignaient naturellement la consommation de viandes et de laitages, etc., dont la production intense tout en étant polluante mobilise l'utilisation de grandes ressources diverses, particulièrement en espaces, eau, fourrages et soins vétérinaires. Omar El Mabrouk, dans l'univers du roman ouvre délibérément, sans état d'âme, la voie de la lente mais inéluctable destruction de la richesse de la terre et du savoir-faire ancestral en neutralisant par la force les paysans : « Nous étions à quelques jours de la récolte, mais les gendarmes d'Omar El Mabrouk refusèrent de nous laisser pénétrer dans les champs » (L'Honneur de la tribu 168). La construction de cette phrase à l'allure simple est révélatrice : la conjonction «mais » porte l'accent sur l'opposition entre ce que veulent les arboriculteurs (accomplir la récolte des olives) et son empêchement par les gendarmes qui agissent pour le compte d'une autorité personnifiée (Omar El Mabrouk, à qui, trivialement, les gendarmes « appartiennent ») et non en tant que force légale de coercition relevant de l'État (même si Omar El Mabrouk en est le représentant le plus notoire en tant que Wali, préfet) que seul le procureur de la République actionnerait dans le cadre d'un dispositif judiciaire n'occultant point les intérêts des paysans de Zitouna. Délégué par le pouvoir central pour mettre en place un embryon administratif qui poserait les jalons d'un processus visant, de concert avec la population, à moderniser progressivement la région, Omar El Mabrouk, faisant fi de l'existence, ne serait-ce que comme simulacre, de «la politique », projette ses fantasmes, ses désirs, ses ressentiments, en des actes aussi néfastes les uns que les autres. Ce sera son fils, qu'il ne connaît pas, fruit de ses rapports incestueux avec sa propre sœur, elle aussi morte en couches durant la guerre de libération nationale après avoir partagé sa vie durant une courte période avec un jeune officier français, chef de poste, devenu avocat qui va l'affronter sur le terrain judiciaire pour défendre la cause des gens de Zitouna. Par conséquent, dans ce roman de l'honneur (sous toutes ses formes) mis à mal, où les idéologies s'affrontent, le transgressif se condense à partir de ses multiples motifs. En même temps, tout en révélant la vacuité de « la politique » dans un État autoritaire, à la source de pouvoir illégitime, le transgressif s'autorise aussi à travers « le politique » dont il revêt les habits, une percée significative ${ }^{13}$. Cette nécessaire mais vaine tentative fera cependant germer quelque chose puisque le vieux narrateur désabusé clôt son histoire ainsi : "Ce récit a réveillé mes souvenirs de jeunesse... Il y a longtemps... Bien longtemps... Je crois bien que j'ai envie de mourir » (L'Honneur de la tribu 216).

\footnotetext{
${ }^{13}$ Le fils d'Omar El Mabrouk qui défie de manière transgressive son père, tente d'établir, entre le pouvoir central et les paysans de Zitouna, un contact.
} 
Pour arriver au plus près de l'articulation du transgressif au politique, je citerai un passage du Fleuve détourné, où quelques lignes en italique ayant pour narrateur Vingt-Cinq, zoophile occasionnel, qui a aussi engrossé sa cousine en dehors du mariage. Ce paragraphe rappelle, indirectement, que les rapports sexuels hors mariage sont prohibés à la fois religieusement et civilement et repoussent ainsi les bornes du transgressif. Effectivement, la section des mœurs existe encore dans les commissariats de police en Algérie où les agents contrôlent les identités pour vérifier le statut familial des couples interpellés dans des lieux publics ou autres. Mimouni a, donc, écrit pour mieux situer le désarroi et la misère sexuels :

\begin{abstract}
'Je profitais de cette proximité pour lui rendre de fréquentes visites nocturnes. Mais il fallait se montrer prudent : l'ânesse comptait de nombreux amants parmi la nuée de cousins. D'un tacite accord, nous étions convenus de ne pas nous dénoncer, le tard-venu devant se défiler sans bruit s'il trouvait l'ânesse occupée'.

'Merveilleuse entente, fondée sur le sexe hospitalier de l'ânesse! Notre système était magnifique, car nul ne songeait à en remettre les options fondamentales. Nous pensions bien gardé le secret de nos conventions, et par conséquent assuré l'approbation populaire.' (41-42, emphase de l'auteur)
\end{abstract}

Dans cette citation, le passage en italique, qui consacre euphoriquement dans un élan transgressif, entre cousins, le partage des faveurs de l'ânesse, en dit long sur le glissement tout aussi transgressif et cinglant de par son ironie voilée, de l'espace microsocial - où se joue le drame de la sexualité, déviante parce que réprimée - à l'espace macrosocial, c'est-à-dire de « la politique » où les options fondamentales, du régime socialiste pour Mimouni, n'ont de résonances qu'internes alors que les jeunes copulent avec des animaux ${ }^{14}$. Le lexique utilisé par l'auteur met en parallèle les deux tragédies en utilisant les mêmes mots montrant par là même que deux discours s'interpénètrent, s'installent dans l'interdiscours qui fait que l'un ne peut se passer de l'autre, mais qu'ils signifient tout autrement le réel. Deux situations d'énonciation où s'insinue l'absurde. Il n'y a que les dirigeants de pays autoritaires qui tiennent des propos tels que «Notre système était magnifique ». De même, «l'approbation populaire» est une construction magique qu'un système rhétorique rodé fait valoir même auprès des opprimés, souvent paralysés par leurs conditions de vie et les promesses dont on les abreuve. Pour Mimouni, les « options fondamentales » du système algérien ne peuvent se révéler que dans leurs incohérences (d'abord en tant que pensée politique de circonstances, sans projet mobilisateur, mais, et surtout dans leur déploiement au quotidien) et les manifestations du transgressif. La proposition qui précède celle énoncée en italique évoque avec un rare voilement stylistique la «complicité »dont font preuve non pas les cousins de filiation, mais ceux qui détiennent le pouvoir, se le transmettent comme un héritage familial. "L'ânesse », comme en métempsychose, abrite l'âme du pays et renvoie au viol à répétitions plus pernicieux, celui permanent de la violence symbolique. Critique, cette citation est aussi pesante en raison de sa charge d'amertume (irradiant l'exultation que dégage la forme du discours) et

\footnotetext{
${ }^{14}$ On ne raille pas la glottopolitique, c'est-à-dire les formules et les slogans qui structurent, paradoxalement par leur vide, même s'ils sont idéologiquement marqués quant à leur origine, les discours des dirigeants.
} 
d'indignation difficilement répressible pour le scripteur lui-même. Le fiasco total, dans L'Honneur de la tribu, de l'inauguration du «souk el fellah », surface de vente, démagogiquement nommée «le marché du paysan », alors qu'il est plus destiné à la horde de fonctionnaires, de policiers et de gendarmes appelés à faire régner l'ordre, est une illustration de la désinvolture avec laquelle sont traitées les affaires publiques. Il caricature ainsi les défauts majeurs de construction de l'édifice infrastructurel et surtout superstructurel de l'État post-colonial ou en voie de développement.

\section{Conclusion}

Dès l'incipit, esthétiquement marqué par un sociolecte propre aux employés des administrations pénitenciaires, du Fleuve détourné, Mimouni donne le ton de la rupture transgressive avec les attente des lecteurs. En effet, la phrase «nos spermatozoides sont subversifs » émanant du héros-narrateur, ancien combattant, annonce que la fécondation du texte va donner naissance aux contestations qui se profilent derrière les voiles de la sexualité, de la religion et du politique. C'est ainsi d'ailleurs que la transgression, un mot que Foucault inscrit, selon Jean-François Favreau, « au principe de la littérature moderne » (2012) occupe fortement l'espace textuel. Dans l'œuvre mimounienne, elle s'érige en un mode fondamental d'énonciation qui prolifère, tel un rizhome aux racines foisonnantes, dans les zones inconnues où se révèle et s'informe ce que l'on pourrait qualifier d' «impossible romanesque ». De même la transgression insuffle une rare énergie aux actants de l'univers fictionnel, tout comme sa plasticité, lui permet d'épouser toutes les formes. Ce qu'il faudrait retenir, en définitive, c'est qu'allant bien au-delà, car, s'appuyant sur une nomenclature invisible, le transgressif revêt les nuances d'une métaphore infinie du politique dans le langage ennemi de la littérature comme art de faire. 


\section{Bibliographie}

Addi, Lahouari. «Forme néo-patrimoniale de l'État et secteur public en Algérie ». Etat et développement dans le monde arabe. Dirigé par H. El-Malki et J.-C. Santucci. Paris : CNRS, 1990.

Ardoino, Jacques. Les avatars de l'éducation. Paris : PUF, 2000.

Arendt, Hannah. Qu'est-ce que la politique ? Paris : Seuil, 1995.

Baechler, Jean. Qu'est-ce que l'idéologie? Paris : Gallimard, 1976.

Charaudeau, Patrick et Dominique Maingueneau. Directeurs. Dictionnaire d'analyse du discours. Paris : Seuil, 2002.

Certeau, Michel de. L'invention du quotidien. Arts de faire 1. Paris : 10/18, 1980.

Danblon, Emmanuelle. «La construction de 1'autorité en rhétorique ». Besançon : Presses Universitaires de Franche-Comté, Semen [en ligne], 2006.

Favreau, Jean-François. Vertige de l'écriture, Michel Foucault et la littérature. Lyon : ENS Éditions. Coll. «Signes », 2012.

Mimouni, Rachid. Le Fleuve détourné. Alger : Laphomic, 1986.

---. Tombéza. Paris : Stock, 2000.

---. L'honneur de la tribu. Alger : Laphomic, 1988.

Tournier, Michel, Vol du vampire. Notes de Lectures. Paris : Mercure de France, 1981. 